\title{
Outcome analysis of biplanar mandibular distraction in adults
}

\author{
Debarati Chattopadhyay, \\ Madhubari Vathulya, \\ Praveen Ambadivalappil \\ Jayaprakash, \\ Akshay Kapoor
}

Department of Burns and Plastic Surgery, All India Institute of Medical Sciences Rishikesh, Rishikesh, India

\begin{abstract}
Background: Mandibular deficiency leading to facial asymmetry causes cosmetic deformity as well as psychological stigma for the patient. Correction of these mandibular asymmetries is a major challenge. The study investigates the efficacy of bidirectional mandible distraction for the treatment of mandibular deficiency.

Methods: This prospective study included six individuals aged between 17 and 24.4 years. Five patients had hemifacial microsomia and one had unilateral temporomandibular joint ankyloses. All patients underwent mandibular distraction osteogenesis. Postoperative skeletal changes in affected mandible, and changes in occlusal plane and oral commissure cant were evaluated using three-dimensional reconstruction. Patient satisfaction and understanding of the procedure were assessed through three questionnaires administered during pre-distraction, distraction and postdistraction phases.

Results: In pre-distraction phase, aesthetic appearance seemed to be the primary indication for surgery. In distraction phase, pain while chewing was the primary handicap. In post-distraction phase all patients were satisfied with the aesthetic outcome. The facial deformity was improved through mandibular distraction osteogenesis. On the affected side in all the patients, height and length of the mandible increased. Canting of the occlusal plane and oral commissure was corrected. Conclusion: Bidirectional mandible distraction is an effective treatment for correction of mandible deformities in adult patients.
\end{abstract}

Keywords: Distraction osteogenesis / Imaging, three dimensional / Mandible / Temporomandibular ankylosis

\section{INTRODUCTION}

Mandibular deficiency is usually caused by the failure of growth of one or more condyles. It may be attributed to congenital de-

Correspondence: Praveen Ambadivalappil Jayaprakash

Department of Burns and Plastic Surgery, All India Institute of Medical Sciences

Rishikesh, flat no 503, Veerbhadra Apartments no 3, Nirmal Bhag, Pashulok,

Rishikesh 249203, India

E-mail: viperpraveen@gmail.com

We thank Dr. Swagata Chowdhury (Chittagong National Cancer Institute, Kolkata, India) for his help in the cephalometric analysis, Ms. Vaishali Verma (Maveric

Solutions Inc, Delhi, India) for her help in 3D imaging and analysis, and Dr. Ankita Kaushik (All India Institute of Medical Sciences, Rishikesh, India) for her help with artwork in figure $1 \mathrm{~B}$.

Received September 23, 2020 / Revised January 20, 2021 / Accepted February 20, 2021 formities, such as hemifacial microsomia, Goldenhar syndrome, Pierre Robin sequence, or Treacher-Collins syndrome [1]. The condition may also be acquired due to trauma, infection, or temporomandibular joint ankylosis. Reconstruction of mandibular deficiencies remains a challenge. Conventional osteotomies and bone grafting are associated with longer stay in hospitals, increased risk of infection, relapse, and discomfort to the patient. Many patients require multiple surgeries [2].

The method of distraction osteogenesis was introduced by Codivilla at the beginning of the 20th century. During 1950s, Ilizarov contributed to the development of the technique by elucidating the biological and mechanical principles in new bone formation. The first successful clinical application of dis- 
traction osteogenesis was reported by McCarthy et al. in the unilateral and bilateral correction of mandibular deficiency in four children. Since then, distraction osteogenesis has been a preferred treatment method for the correction of abnormality in the craniofacial region [3]. It induces the formation of new bone at the vascularized margins of preexisting bones separated by incremental traction, and the process commonly employs distraction devices for the mechanical stretching of bone tissues [4]. Distraction devices have been classified depending on the direction of distraction and the site of application [2]. External osteodistraction devices can be applied unidirectionally, bidirectionally, and three-dimensionally. By contrast, internal or intraoral distractors can be applied only unidirectionally [2]. The choice of device entirely depends on the treatment goal.

In India, mainly due to economic constraints, neglected adult cases of mandibular deficiency present to plastic surgeons. The patients predominantly visit before marriage when the facial appearance becomes utmost important for social acceptance. The goal of reconstruction in these cases remains to be aesthetic appearance rather than a perfect functional reconstruction. In such cases, distraction osteogenesis provides optimal results because of its ability to stretch the overlying deficient soft tissue along with the bone.

Multiplanar distractors are ideal for providing three-dimensional distraction. However, these distractors are proprietary and their cost is a hindrance to poor patients.

Bidirectional distraction osteogenesis was first described by Molina and Ortiz Monasterio in 1994 [5]. Bidirectional distractors can be easily assembled by local technicians and used safely in adult patients. Bidirectional distraction osteogenesis provides a more precise control of the distraction process compared with unidirectional distraction, which means that bidirectional distraction osteogenesis does not require additional autogenous bone grafting [6].

In this study, we describe the application of bidirectional distractors in six adult patients with congenital mandibular deformities and assess the outcomes.

\section{METHODS}

The study was conducted at our institute between 2016 and 2018. The prospective study included six individuals (four women and two men) with a mean age of 20.4 years (range, 17-24.4 years). In total, five patients had hemifacial microsomia and one had unilateral temporomandibular joint ankyloses. All the patients underwent bidirectional mandibular distraction osteogenesis.

An extraoral mandible distractor was used in all the patients (Fig. 1). Two osteotomies were performed on either side of the gonial angle, and two 2-mm pins were placed in each segment. The mean distraction period was 3.1 months (range, 2.5-4 months). The consolidation period was 6 weeks. Intermaxillary elastics were applied to control lateral mandibular movement.

Facial computed tomography was performed preoperatively (T1) and 1 year after completion of treatment (T2). Clinical photographs were taken preoperatively and at 1-year follow-up.

In order to assess patient satisfaction, we used the questionnaire designed by Datta et al. [7] for the pre-distraction, distraction and post-distraction phases. The pre-distraction questionnaire was administered 1 week before surgery after the patient had been counseled regarding the surgery. The distraction phase questionnaire was given to the patient in the third to fourth week after surgery once the patient had entered the consolidation phase. The post-distraction phase questionnaire was filled by the patient 6 months after removal of the distraction device.
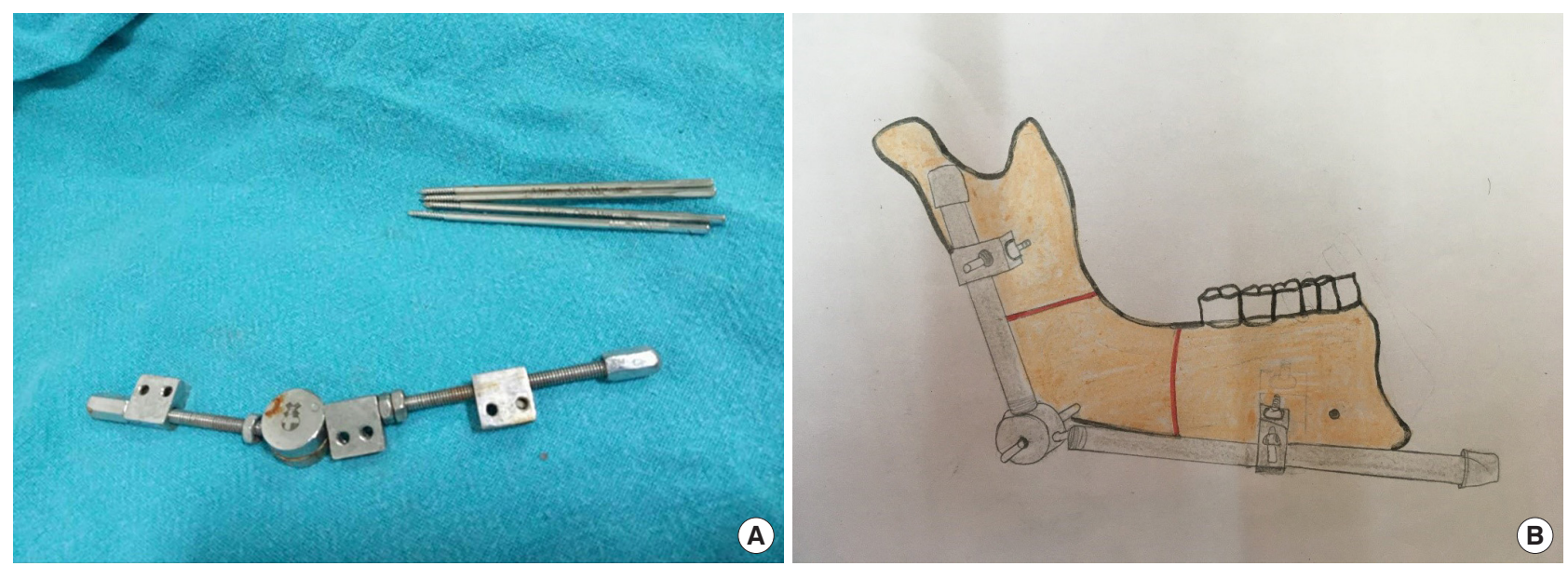

Fig. 1. (A) A biplanar distractor. (B) Schematic drawing showing distractor placement. 
Measurement was performed using Geomagic Freeform software (3D Systems, Rock Hill, SC, USA). Features evaluated were skeletal changes in the affected mandible, changes in the occlusal plane, and changes in oral commissure cant. Three-di-

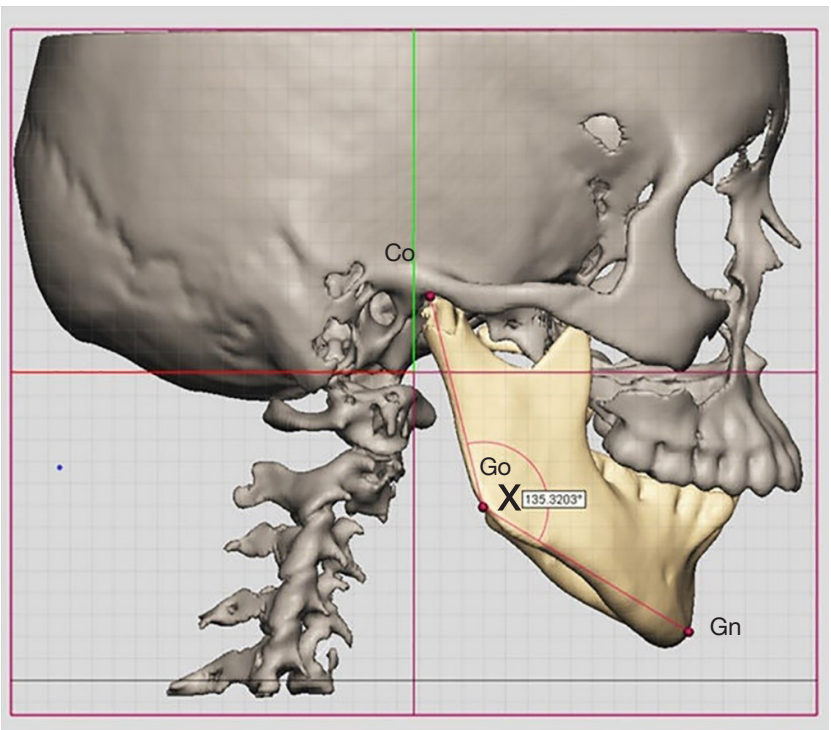

Fig. 2. Lateral view of a three-dimensional reconstructed skull. Co, condylion; Go, gonion, Gn, gnathion; Co-Go, ramus height; Go-Gn, body length; $\mathrm{X}$, gonial angle.

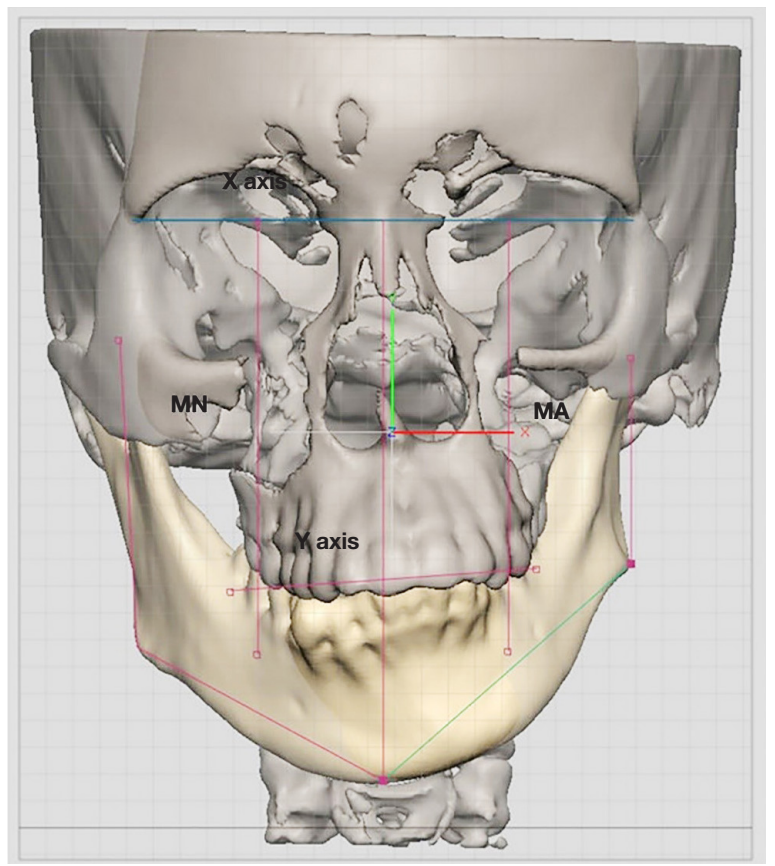

Fig. 3. Occlusal plane. X-axis, line connecting the bilateral lo (latero-orbitale) points; Y-axis, line perpendicular to the X-axis through the center of the skull; MA, vertical distance of maxillary molar to the $\mathrm{X}$-axis on the affected side; $\mathrm{MN}$, vertical distance of maxillary molar to the $\mathrm{X}$-axis on the unaffected side; chin deviation, horizontal distance of chin to the Y-axis (the chin deviating toward the affected side is defined as a negative value). mensional reconstruction was used to observe the skeletal changes in the affected mandible. Both sides of the mandibular contour in the lateral view were seen (Fig. 2).

The parameters that were used to determine changes in the occlusal plane were (Fig. 3): line connecting the bilateral lo (latero-orbitale) points $=\mathrm{X}$-axis; line perpendicular to the $\mathrm{X}$ axis through the center of the skull $=\mathrm{Y}$-axis; $\mathrm{MA}=$ vertical distance of maxillary molar to the $\mathrm{X}$-axis on the affected side; $\mathrm{MN}=$ vertical distance of maxillary molar to the $\mathrm{X}$-axis on the unaffected side; chin deviation = horizontal distance of chin to the Y-axis (the chin deviating toward the affected side is defined as a negative value).

The parameters that were used to determine changes in oral commissure cant were (Fig. 4): measurement of the frontal facial photograph; a line connecting the pupils in both eyes defined as the horizontal reference plane; $F A=$ vertical distance of oral commissure to the horizontal reference plane on the affected side; $\mathrm{FN}=$ vertical distance of oral commissures of the horizontal reference plane on the unaffected side; $F$ ratio $=F / F N$.

\section{RESULTS}

Tables 1-3 depict the changes in parameters, namely Co-Go

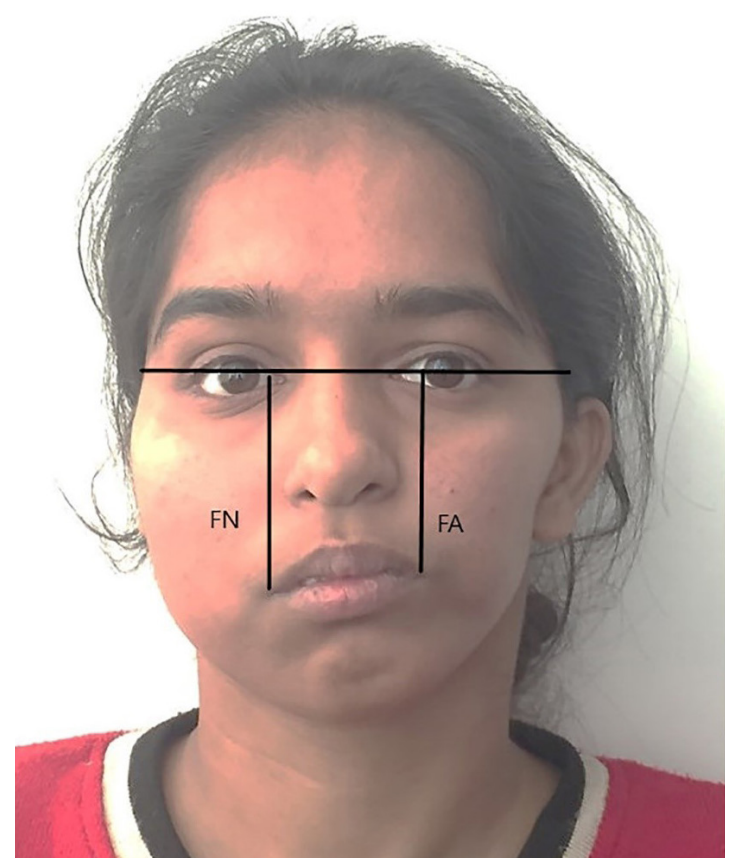

Fig. 4. Oral commissure cant. Measurement of the frontal facial photograph, and mark line connecting the pupils in both eyes defined as the horizontal reference plane. FA, vertical distance of oral commissure to the horizontal reference plane on the affected side; $\mathrm{FN}$, vertical distance of oral commissures of the horizontal reference plane on the unaffected side; $F$ ratio=FA/FN. 
Table 1. Skeletal changes in the mandible

\begin{tabular}{lcc}
\hline \multirow{2}{*}{ Change } & \multicolumn{2}{c}{ Mean (range) } \\
\cline { 2 - 3 } & $51.2(48.5-53.3)$ & $50.4(48.4-52.9)$ \\
\hline Co-Go (N, mm) & $37.4(36.5-39.1)$ & $48.8(46.5-49.7)$ \\
Co-Go (A, mm) & $54.3(53.1-55.3)$ & $53.6(53.0-54.1)$ \\
Go-Gn (N, mm) & $58.5(56.9-59.1)$ & $61.9(58.8-62.2)$ \\
Go-Gn (A, mm) & $142.2(141.8-143.1)$ & $142.1(140.6-143.5)$ \\
Gonial angle (N, $\left.{ }^{\circ}\right)$ & $145.6(144.2-147.5)$ & $143.2(142.2-144.2)$ \\
\hline Gonial angle (A, $\left.{ }^{\circ}\right)$ & & \\
\hline
\end{tabular}

Co, condylion; Go, gonion; Gn, gnathion; Co-Go, ramus height; Go-Gn, body length; $\mathrm{N}$, normal side of the mandible; $\mathrm{A}$, affected side of the mandible; $\mathrm{T1}$, mean measurements before distraction; $\mathrm{T} 2$, mean measurements after distraction.

Table 2. Changes in the occlusal plane

\begin{tabular}{|c|c|c|}
\hline \multirow{2}{*}{ Change } & \multicolumn{2}{|c|}{ Mean (range) } \\
\hline & $\mathrm{T} 1$ & T2 \\
\hline $\mathrm{MN}(\mathrm{mm})$ & $68.1(67.5-69.1)$ & $68.8(67.1-69.9)$ \\
\hline $\mathrm{MA}(\mathrm{mm})$ & $62.7(61.4-62.9)$ & $68.6(67.6-69.1)$ \\
\hline Chin deviation (mm) & -10.88 & -3.67 \\
\hline
\end{tabular}

$M N$, vertical distance of maxillary molar to the $X$-axis on the unaffected side; $M A$ vertical distance of maxillary molar to the $\mathrm{X}$-axis on the affected side; $\mathrm{T} 1$, mean measurements before distraction; $\mathrm{T} 2$, mean measurements after distraction.

Table 3. Change in the oral commissure cant

\begin{tabular}{lcc}
\hline & $\mathrm{T} 1$ & $\mathrm{~T} 2$ \\
\hline F ratio (FA/FN) & 0.86 & 0.96 \\
\hline
\end{tabular}

FA, vertical distance of oral commissure to the horizontal reference plane on the affected side; FN, vertical distance of oral commissures of the horizontal reference plane on the unaffected side; $\mathrm{T} 1$, mean measurements before distraction; $\mathrm{T} 2$, mean measurements after distraction.

(N), Co-Go (A), Go-Gn (N), Go-Gn (A), gonial angle (N), gonial angle $(\mathrm{A}), \mathrm{MN}, \mathrm{MA}$, chin deviation, and $\mathrm{F}$ ratio.

Assessment of the skeletal changes in the mandible revealed an increase in ramus height and body length, whereas a decrease in the gonial angle. On analysis, we found that following distraction, the mean mandibular height represented by Co-Go distance on the affected side, increased by $11.4 \mathrm{~mm}$. The mean mandibular length on the affected side, represented by Go-Gn distance, was found to increase by $3.4 \mathrm{~mm}$. Because of a higher increase in mandibular height as compared to the length we found that the mean gonial angle on the affected side decreased by $2.4^{\circ}$.

Postoperative changes in the occlusal plane and oral commissure cant was also noted in terms of chin deviation and $\mathrm{F}$ ratio, respectively. In order to quantify the change in occlusal cant we used the distance MA which represented the vertical distance of the maxillary molar to the line passing through the lateral orbitale (X-axis) on the affected side. Following distraction, the mean increase in this distance on the affected side was found to
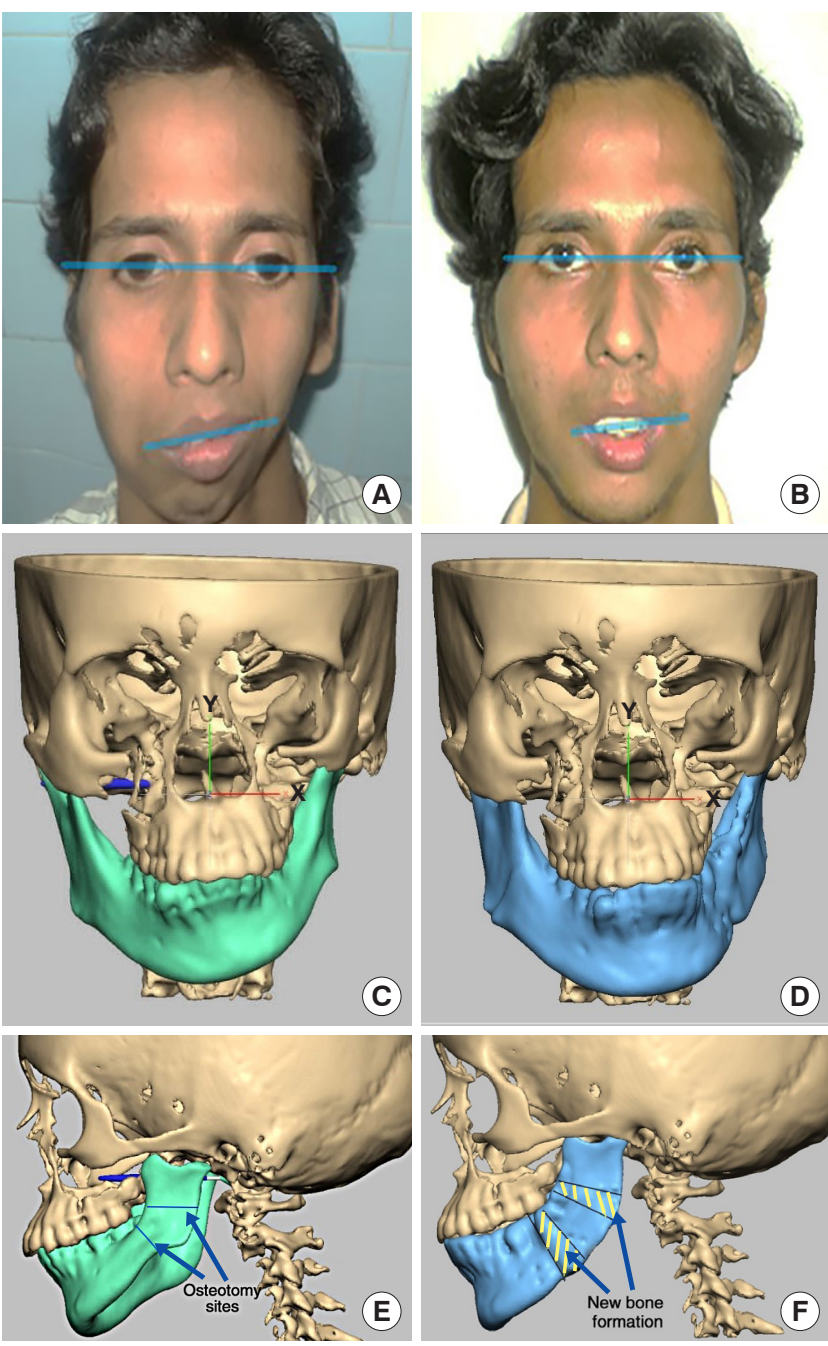

Fig. 5. (A) Preoperative facial profile of an 18-year-old male patient with left hemifacial macrosomia. (B) One-year follow-up picture of the patient. (C) Frontal view of the facial skeleton (preoperative). (D) Postoperative frontal view showing skeletal changes. (E) Lateral view of facial skeleton (blue arrows: preoperative). (F) Postoperative lateral view showing skeletal changes (blue arrows: sites of new bone formation marked). X-axis, line connecting the bilateral lo (lateroorbitale) points; Y-axis, line perpendicular to the $\mathrm{X}$-axis through the center of the skull.

\section{be $5.9 \mathrm{~mm}$.}

The chin deviation was measured as the distance of gnathion from the Y-axis and following distraction the mean shift was measured to be $7.21 \mathrm{~mm}$ from the affected side towards Y-axis.

The change in cant of the oral commissure was determined using the $\mathrm{F}$ ratio, which is the ratio of the vertical distance from $\mathrm{X}$ axis to the oral commissure of the affected side (FA) to the normal side (FN). In a normal face the ratio should be 1. Following distraction, we found that this ratio increased from 0.86 to 0.96 .

The results confirmed that bidirectional distraction osteogenesis increased the height and length of the mandible on the affected side. Canting of the occlusal plane and oral commissure 
Table 4. Evaluation of patient priorities and understanding in the pre-distraction phase

\begin{tabular}{|c|c|c|c|c|c|c|c|c|}
\hline \multirow{2}{*}{ No. } & \multirow{2}{*}{$\begin{array}{c}\text { Sex/age } \\
\text { (yr) }\end{array}$} & \multirow{2}{*}{$\begin{array}{l}\text { Understood } \\
\text { procedure? }\end{array}$} & \multicolumn{3}{|c|}{ Reasons for seeking surgery } & \multirow{2}{*}{ Motivating factor } & \multirow{2}{*}{$\begin{array}{l}\text { Understood all } \\
\text { modalities? }\end{array}$} & \multirow{2}{*}{$\begin{array}{l}\text { Reaction to } \\
\text { device }\end{array}$} \\
\hline & & & Aesthetic & Function & Most Imp & & & \\
\hline 1 & $F / 23$ & Yes & Aesthetic & None & Aesthetic & Self, family & Yes & Acceptable \\
\hline 2 & $F / 22$ & Yes & Aesthetic & Chewing & Aesthetic & Self, family & Yes & Acceptable \\
\hline 3 & $F / 24.4$ & Yes & Aesthetic & Chewing & Aesthetic & Self, family & Yes & Acceptable \\
\hline 4 & $\mathrm{~F} / 17$ & Yes & Aesthetic & None & Aesthetic & Self, family & Yes & Bulky \\
\hline 5 & $\mathrm{M} / 18$ & Yes & Aesthetic & None & Aesthetic & Self, family & Yes & Acceptable \\
\hline 6 & $\mathrm{M} / 19$ & Yes & Aesthetic & None & Aesthetic & Self, family & Yes & Acceptable \\
\hline
\end{tabular}

F, female; M, male.

Table 5. Evaluation of patient comfort during the distraction phase

\begin{tabular}{lllllllll}
\hline No. & $\begin{array}{c}\text { Sex/age } \\
(\text { yr) }\end{array}$ & $\begin{array}{c}\text { Appliance and } \\
\text { expectations }\end{array}$ & Complaints & People's comments & Type of comments & $\begin{array}{c}\text { Response to } \\
\text { comments }\end{array}$ & Suffering? & Right decision \\
\hline 1 & F/23 & Yes & Pain, transient numbness & Always & Encouraging & Ignore & Not sure & Yes \\
2 & F/22 & Yes & Pain & Always & Encouraging & Ignore & Not sure & Yes \\
3 & F/24.4 & Yes & Pain & Always & Neutral & Ignore & Not sure & Yes \\
4 & F/17 & Yes & Pain & Always & Neutral & Ignore & Yes & Yes \\
5 & M/18 & Yes & Pain & Always & Encouraging & Ignore & No & Yes \\
6 & M/19 & Yes & Pain & Sometimes & Encouraging & Ignore & No & Yes \\
\hline
\end{tabular}

F, female; M, male.

Table 6. Evaluation of patient satisfaction in the post-distraction phase

\begin{tabular}{lllllllllll}
\hline No. & $\begin{array}{c}\text { Sex/age } \\
\text { (yr) }\end{array}$ & $\begin{array}{c}\text { Level of } \\
\text { satisfaction }\end{array}$ & Facial app & Speech & Breathing & Chewing & Most important & $\begin{array}{c}\text { Treatment } \\
\text { worth it? }\end{array}$ & $\begin{array}{c}\text { Treatment worth } \\
\text { problems faced? }\end{array}$ & $\begin{array}{c}\text { Would you } \\
\text { recommend? }\end{array}$ \\
\hline 1 & F/23 & Satisfied & Better & No & No & Can't say & Appearance & Yes & Yes & Yes, no hesitation \\
2 & F/22 & Totally satisfied & Better & No & No & Yes & Appearance & Yes & No & Yes, no hesitation \\
3 & F/24.4 & Satisfied & Better & No & No & Yes & Appearance & Yes & Yes & Yes, no hesitation \\
4 & F/17 & Satisfied & Better & No & No & No & Appearance & Yes & Yes & Yes, some hesitation \\
5 & M/18 & Satisfied & Better & No & No & Can't say & Appearance & Yes & Yes & Yes, no hesitation \\
6 & M/19 & Satisfied & Better & No & No & No & Appearance & Yes & yes & Yes, no hesitation \\
\hline
\end{tabular}

F, female; M, male.

was corrected. Overall, the facial deformity was corrected.

In Fig. 5, the representative case of left-sided hemifacial microsomia is presented to depict the facial and skeletal changes after unilateral bidirectional distraction osteogenesis.

With regard to indication of surgery in the pre-distraction phase (Table 4) all the patients said their main concern was problem with the facial appearance as the motivating factor for surgery with the family also playing a major role in motivating the patients for surgery. Two out of six patients (33\% of the study group) also complained of problem in chewing as another factor for undergoing surgery. All Patients understood the treatment protocol and were willing to undergo distraction with an extraoral device.

In the distraction phase (Table 5) pain and difficulty in chewing were the major complications faced by the patients. One patient complained of transient numbness of lower lip but it sub- sequently resolved. In the post-distraction phase (Table 6) all patients were happy with the esthetic outcome of the procedure and came to the conclusion that they would definitely recommend the procedure to other patients facing similar problems.

\section{DISCUSSION}

Reconstruction of mandibular asymmetry in adults is extremely difficult. Many procedures exist to correct facial asymmetry in these patients, such as mandibular and maxillary osteotomies with/without bone grafting [5]. These procedures are usually associated with long hospital stay, morbidity, chances of infection, and relapse. Orthognathic surgery for mandibular deficiency in adults is also difficult because of the overlying soft tissue deficiency [8].

Distraction osteogenesis has proven to be a safe and effective 
alternative in the management of craniofacial deficiencies. It induces the formation of new bones at the vascularized margins of preexisting bones separated by incremental traction. As a natural consequence, the overlying soft tissues are also stretched. It reduces the requirement of bone grafts. Distraction osteogenesis can be performed at any age, and when performed in adults after completion of skeletal growth, chances of relapse are less.

In our patients, distraction osteogenesis was performed using external biplanar distractors. Double osteotomy was performed in each case and distractors were applied to achieve both vertical and horizontal lengthening. Contraindications to double osteotomy include small children and inadequate bony stock [2]. All our patients were adults with complete bone growth. With careful preoperative planning, risk of avascular necrosis of the intermediate bone block can be minimized. In our series, there was no avascular necrosis.

Although multiplanar distraction would have been ideal for mandibular distraction osteogenesis in adults, the devices are proprietary and often out of reach of poor patients. Therefore, we used the biplanar distractor, which can be easily made locally and is affordable. Our rationale for using this device was since our patients were all adults and had come for aesthetic correction, an increase in height and length of mandibles would effectively camouflage the facial deformity.

We used three-dimensional reconstruction to analyze our results. In the recent years, three-dimensional skeletal analysis has proven to be equally accurate for cephalometric analysis [9]. In our patients, facial scanning was performed preoperatively, and 6 months and 1 year postoperatively. Computed tomography scanning was performed preoperatively and 1 year postoperatively. Geomagic Freeform software was used to analyze and calculate the results.

The prime factor for motivation for surgery was the aesthetic appearance and the decision was influenced by family members in $100 \%$ of cases. In the study of Datta et al. [7] the family members were involved in $58.3 \%$ of the cases in motivating the patient for surgery. Since all patients in our study group were young and not married so the family had a much more prominent role in decision-making.

In Datta et al. [7] about $53.84 \%$ of the study group readily accepted the extraoral appliance, while the others had some apprehension regarding the bulk and esthetics. In our study group, $100 \%$ of the study group accepted the extraoral device. One of the reasons for this change in attitude towards the extraoral device was probably the financial constraint faced by all patients in our study group.

The most common side effect of the distraction phase was reported as pain in the lower jaw and difficulty in chewing
$[7,10,11]$. Although most patients in our study group complained of pain, it was managed with oral dose of NSAIDs. Only two out of six patients in the study group complained of difficulty in chewing during the distraction phase. But the rate of patients experiencing transient numbness of the lower lip varied widely in all studies. While Datta et al. reported 76\% patients experiencing this complication, only $14 \%$ had transient numbness in the study group of Primrose et al. [7,11]. In our group $16 \%$ of patients experienced this complication. Regarding social interaction most of our patients preferred to avoid social contact, as most people were inquisitive about their device. Although they found encouragement after they explained the procedure, still they felt uncomfortable in talking about it. This phenomenon was also reported in studies by Primrose et al. [11] and Pelo et al. [12] the where a majority of their patients admitted to socializing less and feeling "abnormal," self-conscious and uncomfortable in public situations.

In the post-distraction phase $16 \%$ of our patients were "totally satisfied" while $84 \%$ said they were "satisfied" with the procedure. This was similar to results by Datta et al. [7] where 30\% were "totally satisfied" and $69 \%$ were "satisfied" with the procedure.

One of the important measures of patient satisfaction is whether they would undergo the treatment again considering the problems they faced. One hundred percent of our study group said they found the treatment worth the problems faced and would definitely recommend it to other patients. Similar results were shown by Datta et al. [7] and Ayoub et al. [10] who found $84.61 \%$ and $78.57 \%$ of study groups would recommend this procedure to other patients without any hesitation.

The cases presented in our study are unique because of various reasons. First, biplanar mandibular distraction has been used for correction of mandible deficiency in adult patients. There is paucity of literature in this regard because neglected cases present more frequently in developing countries and the patients are usually managed at an earlier age in developed countries. Our aim was to provide optimum results at minimum cost, and this distractor proved to be effective. Second, bidirectional distractors increased the height and length of the mandible, effectively camouflaging the facial deformity. Because no face is perfect, the patients were satisfied with the improved facial profile. Third, three-dimensional reconstructionbased outcome analysis was performed. Similar studies with multiplanar distractors have documented the results based on cephalometric findings [13]. We found no studies using biplanar distractors where three-dimensional scanning was used to measure the outcome. With this technology, we could prove conclusively that there is indeed an increase in height and 
length of the mandible as well as an improvement in occlusal cant. Overall, the results suggest the efficacy of bidirectional mandibular distraction in the correction of mandibular asymmetry.

In conclusion, mandibular asymmetry in adults creates significant aesthetic concern. With careful surgical planning, complications can be minimized. Bidirectional mandibular distraction remains a safe and viable option for correcting facial symmetry in adults. It is an effective treatment for the correction of mandible deformities in adult patients.

\section{NOTES}

\section{Conflict of interest}

No potential conflict of interest relevant to this article was reported.

\section{Ethical approval}

The study was approved by the Institutional Review Board of All India Institute of Medical Sciences, Rishikesh (IRB No. AIIMS/IEC/17/168) and performed in accordance with the principles of the Declaration of Helsinki. Written informed consent was obtained.

\section{Patient consent}

The patients provided written informed consent for the publication and the use of their images.

\section{ORCID}

Debarati Chattopadhyay

https://orcid.org/0000-0002-0167-7561

Madhubari Vathulya https://orcid.org/0000-0002-7050-5990

Praveen Ambadivalappil Jayaprakash https://orcid.org/0000-0001-8486-5551

Akshay Kapoor

https://orcid.org/0000-0003-4223-9039

\section{Author contribution}

Conceptualization: DC. Methodology: DC, MV, AK, PAJ. Formal analysis: DC. Project administration: DC, MV. Visualization: DC. Writing - original draft: DC. Writing - review and editing: DC, AK, PAJ. Approval of final manuscript: all authors.

\section{REFERENCES}

1. Baskaran M, Arularasan SG, Divakar TK, Thirunavukkarasu R.
Treatment of micrognathia by intraoral distraction osteogenesis: a prospective study. Ann Maxillofac Surg 2017;7:37-44.

2. Maull DJ. Review of devices for distraction osteogenesis of the craniofacial complex. Semin Orthod 1999;5:64-73.

3. Ortakoglu K, Karacay S, Sencimen M, Akin E, Ozyigit AH, Bengi O. Distraction osteogenesis in a severe mandibular deficiency. Head Face Med 2007;3:7.

4. Prabhat KC, Maheshwari S, Gupta ND, Verma SK, Goyal L. Periodontal ligament distraction: a simplified approach for rapid canine retraction. J Indian Soc Periodontol 2012;16:123-5.

5. Molina F, Ortiz Monasterio F. Mandibular elongation and remodeling by distraction: a farewell to major osteotomies. Plast Reconstr Surg 1995;96:825-42.

6. Schleier P, Wolf C, Siebert H, Shafer D, Freilich M, Berndt A, et al. Treatment options in distraction osteogenesis therapy using a new bidirectional distractor system. Int J Oral Maxillofac Implants 2007;22:408-16.

7. Datta R, Utreja A, Singh SP, Rattan V. Satisfaction audit of patients undergoing mandibular distraction osteogenesis with extra-oral distraction appliances. J Maxillofac Oral Surg 2015; 14:212-8.

8. Yamauchi K, Takahashi T. Maxillary distraction osteogenesis combined with mandibular osteotomy to correct asymmetry of the maxillomandibular complex. Plast Reconstr Surg 2006; $118: 39 \mathrm{e}-45 \mathrm{e}$

9. Yitschaky O, Redlich M, Abed Y, Faerman M, Casap N, Hiller N. Comparison of common hard tissue cephalometric measurements between computed tomography $3 \mathrm{D}$ reconstruction and conventional 2D cephalometric images. Angle Orthod 2011;81 11-6.

10. Ayoub AF, Duncan CM, McLean GR, Moos KF, Chibbaro PD. Response of patients and families to lengthening of the facial bones by extraoral distraction osteogenesis: a review of 14 patients. Br J Oral Maxillofac Surg 2002;40:397-405.

11. Primrose AC, Broadfoot E, Diner PA, Molina F, Moos KF, Ayoub AF. Patients' responses to distraction osteogenesis: a multi-centre study. Int J Oral Maxillofac Surg 2005;34:238-42.

12. Pelo S, Gasparini G, Di Petrillo A, Tamburrini G, Di Rocco C. Distraction osteogenesis in the surgical treatment of craniostenosis: a comparison of internal and external craniofacial distractor devices. Childs Nerv Syst 2007;23:1447-53.

13. Ko EW, Hung KF, Huang CS, Chen PK. Correction of facial asymmetry with multiplanar mandible distraction: a one-year follow-up study. Cleft Palate Craniofac J 2004;41:5-12. 